ARTICLE

Received 12 Sep 2016 | Accepted 23 Feb 2017 | Published 20 Apr $2017 \quad$ DOl: 10.1038/ncomms15048 OPEN

\title{
Chytrid fungus infection in zebrafish demonstrates that the pathogen can parasitize non-amphibian vertebrate hosts
}

\author{
Nicole Liew ${ }^{1,2}$, Maria J. Mazon Moya ${ }^{1}$, Claudia J. Wierzbicki², Michael Hollinshead ${ }^{3}$, Michael J. Dillon ${ }^{4}$, \\ Christopher R. Thornton ${ }^{4}$, Amy Ellison ${ }^{5}$, Jo Cable ${ }^{5}$, Matthew C. Fisher ${ }^{2, \star} \&$ Serge Mostowy ${ }^{1, \star}$
}

Aquatic chytrid fungi threaten amphibian biodiversity worldwide owing to their ability to rapidly expand their geographical distributions and to infect a wide range of hosts. Combating this risk requires an understanding of chytrid host range to identify potential reservoirs of infection and to safeguard uninfected regions through enhanced biosecurity. Here we extend our knowledge on the host range of the chytrid Batrachochytrium dendrobatidis by demonstrating infection of a non-amphibian vertebrate host, the zebrafish. We observe dose-dependent mortality and show that chytrid can infect and proliferate on zebrafish tissue. We also show that infection phenotypes (fin erosion, cell apoptosis and muscle degeneration) are direct symptoms of infection. Successful infection is dependent on disrupting the zebrafish microbiome, highlighting that, as is widely found in amphibians, commensal bacteria confer protection against this pathogen. Collectively, our findings greatly expand the limited tool kit available to study pathogenesis and host response to chytrid infection.

\footnotetext{
${ }^{1}$ Section of Microbiology, MRC Centre of Molecular Bacteriology and Infection (CMBI), Imperial College London, London SW7 2AZ, UK. ${ }^{2}$ Department of Infectious Disease Epidemiology, Imperial College London, London W2 1PG, UK. ${ }^{3}$ Division of Virology, Department of Pathology, Cambridge University, Cambridge CB2 1QP, UK. ${ }^{4}$ College of Life and Environmental Sciences, Geoffrey Pope Building, University of Exeter, Exeter EX4 4QD, UK. ${ }^{5}$ School of Biosciences, Cardiff University, Cardiff CF10 3AX, UK. * These authors contributed equally to this work. Correspondence and requests for materials should be addressed to M.C.F. (email: matthew.fisher@imperial.ac.uk) or to S.M. (email: s.mostowy@imperial.ac.uk).
} 
P athogens that exhibit a broad host range constitute a growing threat to biodiversity. This owes to their intrinsic ability to undergo range expansions through humanmediated long-distance dispersal, then spill over and cause subsequent infection of naive hosts ${ }^{1,2}$. Pathogenic fungi exhibit the broadest spectrum of host ranges known for any group of pathogens, with aquatic chytrid fungi in the genus Batrachochytrium emerging as an extinction threat to amphibian species worldwide ${ }^{1,3}$. Two species of amphibian parasitizing chytrids have thus-far been described, Batrachochytrium dendrobatidis $(B d)$ and Batrachochytrium salamandrivorans (Bsal); of these $B d$ is known to parasitize all three orders of amphibians with nearly 700 species of amphibian infected to date $^{4}$. Infection by Bsal thus far appears to be limited to caudates (salamanders) with fewer than 20 species known to be infected ${ }^{5}$.

The mechanisms underlying the ultra-generalist nature of $B d$ compared to its more host-restricted sister species have not yet been described. However, they likely include the ability to deeply invade and establish within the epidermal cells of the stratum corneum through lytic processes and to modulate host immunity through secreted factors ${ }^{6,7}$. While $B d$ infection was originally assumed to be restricted to amphibians, other non-amphibian hosts are thought to sustain infections. Key to this argument are studies showing that crayfish tissues are parasitized by $B d$ in nature, that these infections cause crayfish mortality, and that the infection could be transmitted to co-housed amphibians ${ }^{8}$. The nematode Caenorhabditis elegans is thought to also be parasitized by $B d$ with attendant mortality, and the keratin-rich toenails of waterbirds may act as a transient substrate for $B d^{9,10}$. However, more recent research finds no evidence for roles of non-vertebrate hosts in sustaining infections ${ }^{11}$. An unexamined factor that may confound all of these studies is whether commensal bacteria either interact synergistically with $B d$ to exacerbate infection through dysbiosis, or rather protect against infection through the production of antimicrobial compounds ${ }^{12}$. A microbial role in defining host range is likely to be important as an increasing number of studies are now showing associations between bacterial communities and $B d$ infections of amphibians in the field ${ }^{13-15}$. Taken together, these studies are important as they show that we are missing significant aspects of the biological interactions that define the host range of $B d$.

A thus-far unexamined aspect of $B d$ 's epidemiology is its potential ability to infect other aquatic vertebrates, principally freshwater fish. If $B d$ were able to successfully parasitize fish, then this would represent an unparalleled opportunity for studying infection through use of the widely available zebrafish (Danio rerio). Recent studies have uncovered host immune responses of $B d$ infection in amphibians, though most of this work focused on adaptive immunity despite research suggesting an important role of the innate immune system, especially at the early stages of infection $^{16-21}$. The fully developed innate immune system present in zebrafish larvae, along with its natural transparency, make it possible to easily study host-pathogen interactions in real time using non-invasive live-cell microscopy ${ }^{22-25}$. Furthermore, the immediate environment of the zebrafish larvae can easily be manipulated with antibiotics, allowing the role of commensal bacteria in disease dynamics to be studied. Thus, a zebrafish larvae infection model would present a novel opportunity to study pathogenesis and innate immune responses during $B d$ infection in vivo.

Here we tested whether zebrafish larvae can act as carriers of $B d$ by developing a 3-day dose-dependent model in which infection can be detected and quantified. We show that different stages of $B d$ infection can be observed on zebrafish larvae using histopathology and confocal microscopy, alongside symptoms of infection that are comparable to those observed in amphibians.
These results show that zebrafish larvae undergo an infection process similar to that of $B d$ 's native amphibian hosts, highlighting the utility of zebrafish larvae as an important model to study this emerging panzootic infectious disease.

\section{Results}

$\boldsymbol{B} \boldsymbol{d}$ infection of zebrafish larvae. To investigate if zebrafish larvae at three days post fertilization (d.p.f.) can be infected with $B d$ zoospores (that is, the infectious stage of $B d$ ), a bath water infection model was developed (Supplementary Fig. 1a). To investigate infection burden, we quantified the amount of $B d$ DNA on infected larvae using quantitative PCR (qPCR), an assay typically used to detect $B d$ infection in vivo using swabs or toe clips $^{18,26}$. Initial experiments clearly showed an important role of commensal bacteria in mitigating against $B d$ 's ability to infect larvae. In the presence of antibiotics, intensities of infection were significantly greater when compared to larvae without antibiotic treatment (Fig. 1a, Supplementary Fig. 1b). Subsequently, all further experiments on zebrafish larvae were carried out in the presence of antibiotics. Samples tested show significantly higher genomic equivalents (GE) of $B d$ DNA on $B d$-infected larvae compared to their bath water alone. We observed significantly higher GE of $B d$ DNA on larvae infected with $>200$ zoospores per $\mu \mathrm{l}$ (zsp per $\mu \mathrm{l}$; herein referred to as high dose) of $B d$ zoospores when compared to those infected with $<200$ zsp per $\mu$ l (herein referred to as low dose) of $B d$ zoospores, suggesting a dosedependent nature of infection (Fig. 1b, Supplementary Fig. 1c) We performed qPCR time-course studies and detected $B d$ DNA on larvae up to $96 \mathrm{~h}$ post infection (h.p.i.; Fig. 1c; Supplementary Fig. 1d). To test whether we could extend our findings to include other species of fish, juvenile ornamental guppies (Poecilia reticulata) were infected with $B d$ zoospores. A qPCR timecourse study performed on these guppies showed significantly higher GE of $B d$ DNA on guppies infected with live zoospores compared to those infected with the same dose of heat-killed zoospores at 5 days post infection (d.p.i.; Supplementary Fig. 1e). However, the GE of $B d$ DNA in live zoospore treated guppies became increasingly similar to control values at later time points (12 and 19 d.p.i., respectively), suggesting that infection is unable to persist in this host.

We observed that zebrafish larvae infected with a low dose of $B d$ zoospores show $100 \%$ survival, whereas larvae infected with a high dose of $B d$ zoospores show significant mortality from 0 to 72 h.p.i., supporting that infection is dose dependent (Fig. 1d; Supplementary Table 1). To visualize $B d$ infection in larvae we performed histopathology using methods commonly employed to confirm infection in amphibians ${ }^{27}$. We observed different life-history stages of $B d$ on infected larvae, including mature zoosporangia (that is, the reproductive stage of $B d$ ) and empty discharged sporangia with a discharge tube protruding out of infected tissue. These images prove that $B d$ is able to both encyst and proliferate within the skin of zebrafish larvae (Fig. 1e), a finding that is consistent with the increased detection of GE of $B d$ by qPCR on infected larvae up to 96 h.p.i. The morphology of these $B d$ life-history stages are consistent with those found widely on $B d$-infected amphibians, such as Xenopus laevis ${ }^{7,28}$. Taken together, these results show that zebrafish larvae can be infected with $B d$ in the presence of broad spectrum antibiotics, and that $B d$ infection of larvae resembles that of amphibians.

Symptoms of $\boldsymbol{B} \boldsymbol{d}$ infection in zebrafish larvae. $B d$ infection in a wide range of amphibians can be accompanied by hyperplasia, hyperkeratosis of the skin, lethargy and loss of righting reflex ${ }^{29}$. Infected larvae showed erosion of tail fin, disruption of smooth muscle striations and blistering of skin at 72 h.p.i. 
a
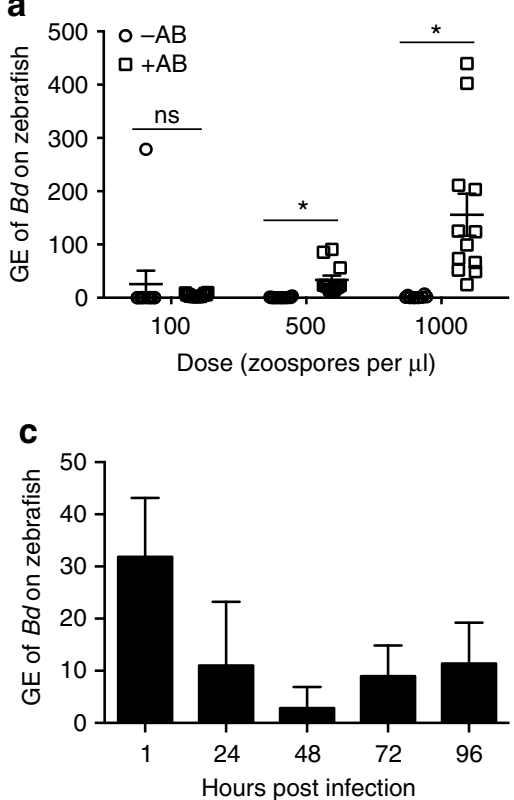

b

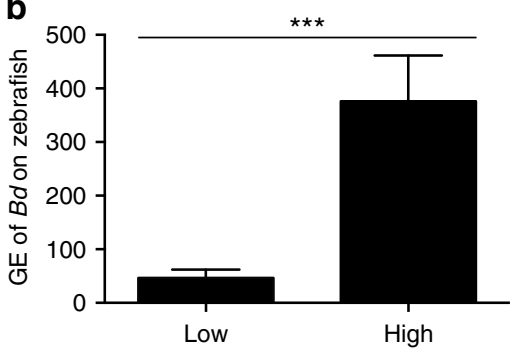

d

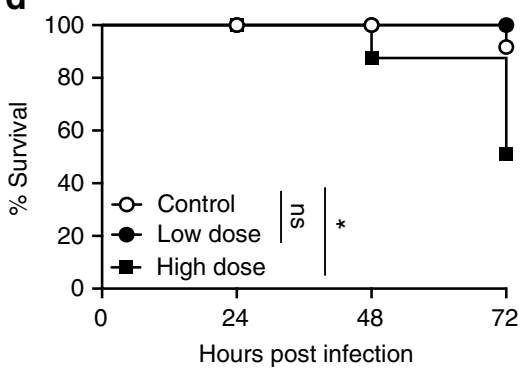

e
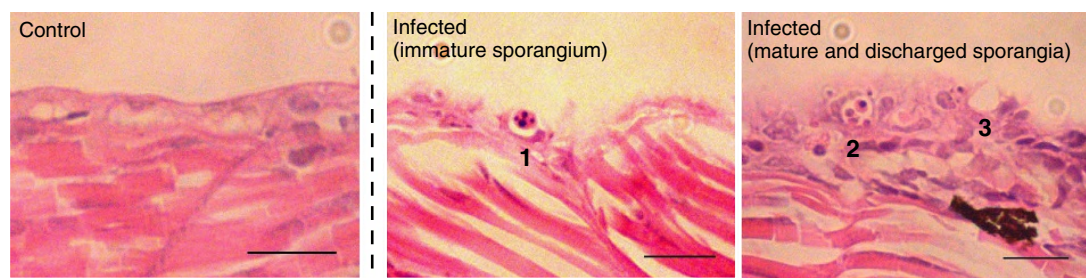

Figure 1 | Bd infection of zebrafish larvae. (a) Zebrafish larvae bath water without ( $-A B$, open circles) or with ( $+A B$, open squares) $1 \%$ penicillin/ streptomycin was inoculated with three doses of $B d$ zoospores. Bath water changed at 24 h post infection (h.p.i.) and larvae incubated for 72 h.p.i. Zebrafish DNA was extracted and amplified by qPCR. Data from 1 experiment shown, using $n=12$ per treatment. Mean \pm s.e.m. are shown. Significance testing performed using unpaired Student's $t$-test (two tailed), ns $P>0.05,{ }^{\star} P<0.05$. See Supplementary Fig. 1b for replicate experiments. (b) Zebrafish larvae bath water was inoculated with low $(<200$ zsp per $\mu$ l) or high ( $>200$ zsp per $\mu$ l) dose Bd zoospores and incubated for 72 h.p.i. Zebrafish DNA was extracted and amplified by qPCR. Data from two experiments plotted here, using $n=12$ per treatment. Mean \pm s.e.m. are shown. Significance testing performed using unpaired Student's $t$-test (two tailed), ${ }^{\star \star \star} P<0.001$. See Supplementary Fig. 1c for replicate experiments. (c) Zebrafish larvae bath water was inoculated with low dose $B d$ zoospores and incubated for 1, 24, 48, 72 or 96 h.p.i. Zebrafish DNA was extracted as in a; Data from one experiment are plotted here (dose $=80 \mathrm{zsp}$ per $\mu \mathrm{l}$ ), using $n=3$ per time point. Mean \pm s.e.m. are shown. See Supplementary Fig. 1d for replicate experiments. (d) Survival curve of zebrafish larvae bath water inoculated with mTGhL (control, open circles, $n=420$ ), low (filled circles, $n=84$ ) or high (filled squares, $n=336$ ) dose $B d$ zoospores. Larvae were incubated for 72 h.p.i. Data pooled from twenty-five experiments. Mean \pm s.e.m. are shown. Significance testing performed by Mantel-Cox (log-rank) test. ns $P>0.05$, ${ }^{\star} P<0.05$. See Supplementary Table 1 for replicate experiments. (e) Zebrafish larvae bath water was inoculated with control or high dose $B d$ zoospores and incubated for 72 h.p.i. Representative longitudinal histological sections shown here. 1, encysted zoosporangium; 2, mature sporangium with internal zoospores; 3 , empty zoosporangium with discharge tube protruding out of epithelial cells exhibiting extensive hyperplasia. Scale bars, $20 \mu \mathrm{m}$. GE, genomic equivalents.

(Supplementary Fig. 2a). To test whether these phenotypes are dose dependent, fin erosion was quantified by measuring two different widths of zebrafish larva caudal tail fin at 72 h.p.i. (Fig. 2a). When compared against control larvae, low or high dose infected larvae showed a $1.7 \pm 0.3$ - or $7.3 \pm 3.5$-fold decrease in dorsal fin length, and a $1.6 \pm 0.1$ - or $7.1 \pm 2.7$-fold decrease in ventral fin length, respectively. To investigate the onset of these infection phenotypes, time-course imaging experiments were performed and showed that morphological changes appeared between 48 and 72 h.p.i. (Fig. 2b). To test whether these phenotypes were caused by a factor associated with increased organic matter or zoospore secreted toxins in $B d$-infected water, we performed time-course imaging studies with exposure of larvae to heat-killed $B d$ zoospores or $B d$ zoospore supernatant. Both treatments showed fin erosion similar to control larvae at all time points tested (Supplementary Fig. 2b,c). These results suggest that fin erosion is largely a consequence of $B d$ infection, although the precise roles of pathogen invasion and/or release of cytolytic factors from adherent $B d$ in establishing this phenotype remains to be determined.

A hallmark of chytrid infection in amphibians includes the presence of zoosporangia and rhizoid-like structures in the keratinized epidermal tissue layers of host skin ${ }^{28,30}$. To investigate symptoms of zebrafish larvae infection, $B d$-infected larvae were labelled with calcofluor white (CFW). We first observed that CFW clearly labelled the chitinous cell wall of $B d$ sporangia in broth culture (Supplementary Fig. 2d). At 72 h.p.i., infected larvae showed fluorescent punctae (bright round spots) throughout the entire body and fin (Fig. 2c). Time-course imaging experiments showed that these phenotypes appeared within 48-72 h.p.i. (Fig. 2c), consistent with the appearance of fin erosion (Supplementary Fig. 2c). We quantified the number of CFW-positive punctae on each larva and observed that larvae infected with a high dose of $B d$ zoospores show significantly more 

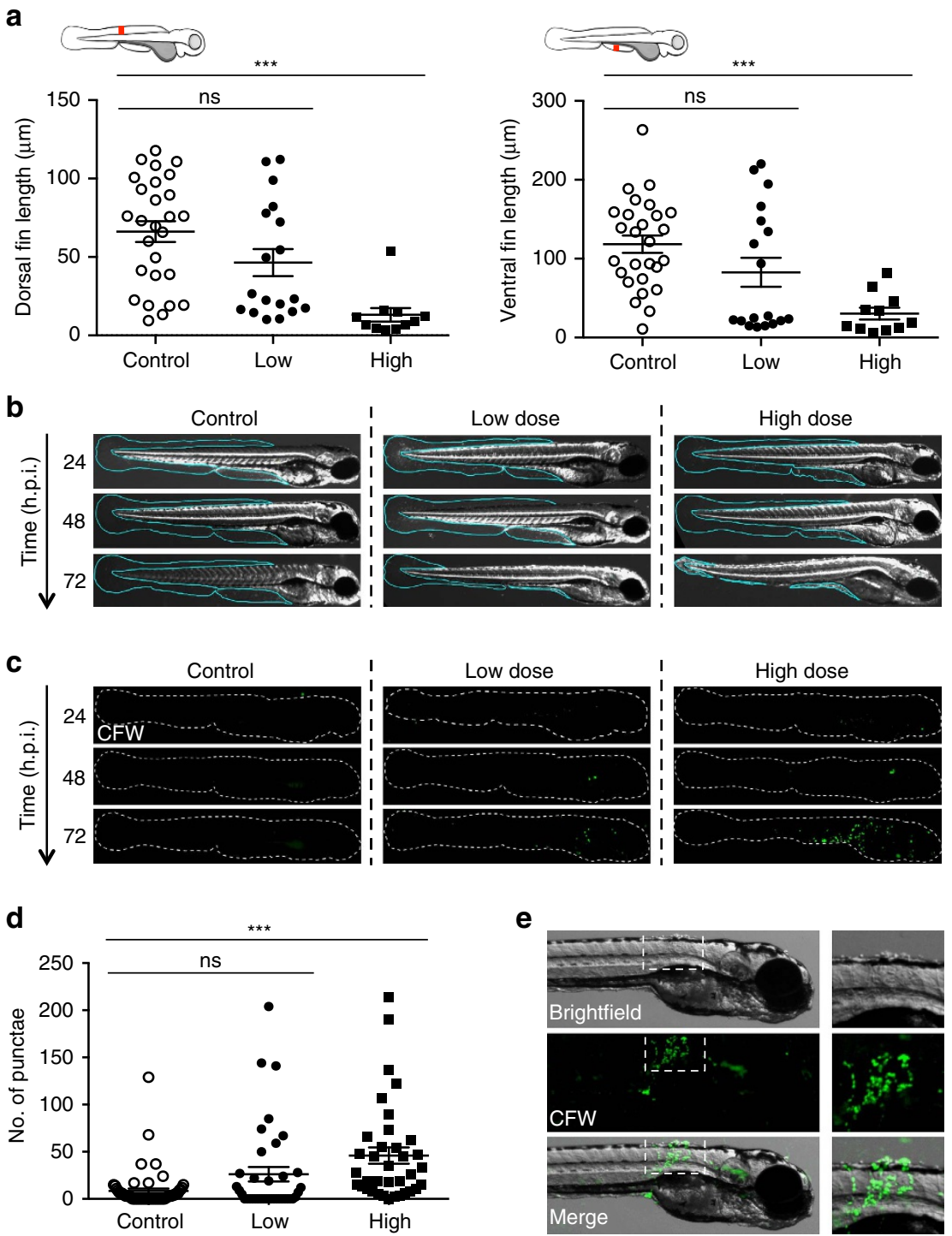

Figure 2 | Symptoms of $\boldsymbol{B d}$ infection in zebrafish larvae. (a) Zebrafish larvae bath water was inoculated with mTGhL plate washings (control, open circles), low ( $<200 \mathrm{zsp}$ per $\mu$ l, filled circles) or high ( $>200 \mathrm{zsp}$ per $\mu$ l, filled squares) dose $B d$ zoospores. Larvae were incubated for 72 h.p.i., where dorsal and ventral fin length was measured (as depicted by red line in cartoon above each graph). Each point represents an individual larva. Data pooled from three experiments per dose, using $n=4-12$ per treatment. Mean \pm s.e.m. are shown. Significance testing performed using unpaired Student's $t$-test (two-tailed), ns $P>0.05,{ }^{\star \star \star} P<0.001$. (b) Zebrafish larvae bath water was inoculated with control, low or high dose $B d$ zoospores, incubated for 72 h.p.i. and imaged by stereomicroscopy. Representative images with cyan outline showing presence or erosion of fin over time. (c) Zebrafish larvae bath water was inoculated with control, low or high dose Bd zoospores and incubated for 24, 48 or 72 h.p.i., then labelled with calcofluor white (CFW; for chitin, green) and imaged by fluorescent stereomicroscopy. Representative images with dotted outline of larvae showing (CFW)-labelled punctae on infected larvae. (d) Enumeration of CFW-labelled punctae from zebrafish larvae whose bath water was inoculated with control (open circles), low (filled circles) or high (filled squares) dose $B d$ zoospores and incubated for 72 h.p.i. Each point represents an individual larva. Data pooled from three experiments per dose, using $n=12$ per treatment. Mean \pm s.e.m. are shown. Significance testing performed using Mann-Whitney test (two tailed), ns $P>0.05,{ }^{\star \star \star} P<0.001$. See also Supplementary Fig. 2e for replicate experiments. (e) Zebrafish larvae bath water was inoculated with high dose Bd zoospores and incubated for 72 h.p.i., then labelled with CFW (green) and imaged by fluorescent stereomicroscopy. Representative images with insets showing colocalization of CFW-labelled punctae with blisters.

(4.7 \pm 0.6 -fold) CFW-positive punctae compared to controls (Fig. 2d, Supplementary Fig. 2e). Larvae infected with a low dose of $B d$ zoospores show similar values to controls, highlighting the dose dependent nature of infection. Moreover, CFW-positive punctae colocalized with blister-like structures on eroded muscle of infected larvae (Fig. 2e), suggesting that both CFW-positive punctae and blistering of skin are symptoms of infection by $B d$.

Consequence of $B d$ infection on zebrafish larvae host tissue. We used confocal microscopy to investigate the consequence of
$B d$ infection on zebrafish larvae. $B d$-infected larvae were fluorescently labelled with CFW and Evans Blue (EB) to detect tissue damage. CFW-positive punctae were observed on the fins and muscle of low dose infected larvae, which colocalize with EB-positive tissue damage (Fig. 3a, Supplementary Fig. 3a). Moreover, both CFW-positive punctae and EB-positive tissue damage were found to colocalize with fin erosion and blister-like structures on $B d$-infected larvae (Supplementary Fig. 3b).

Recent studies have shown that exposure to $B d$ results in apoptosis of amphibian cells in vitro ${ }^{6,31}$. To test whether 
a
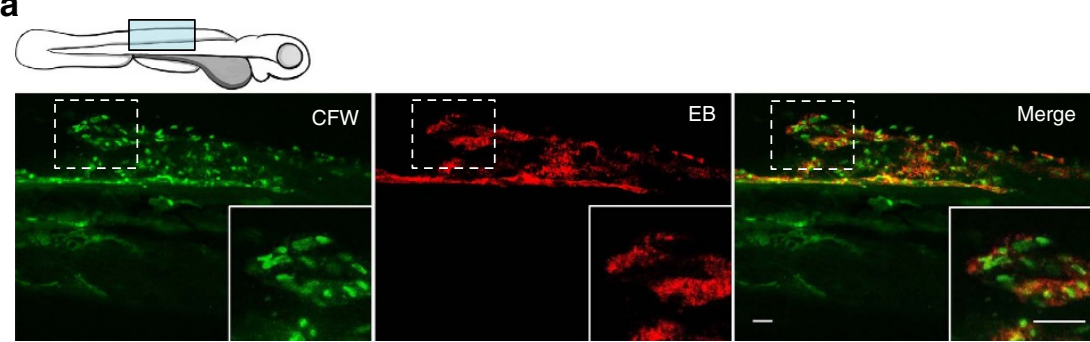

b
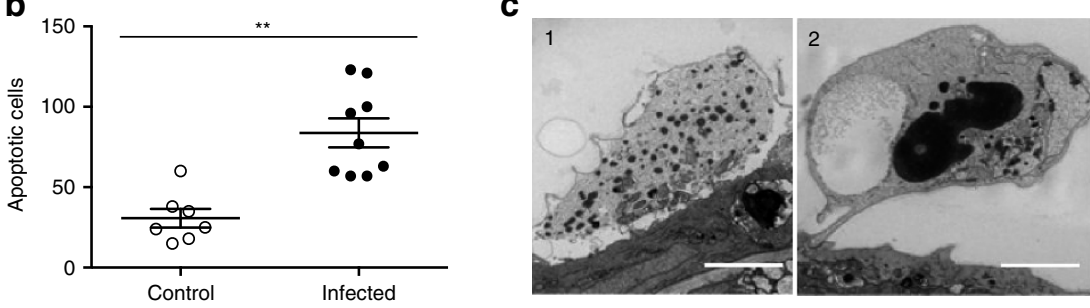

d

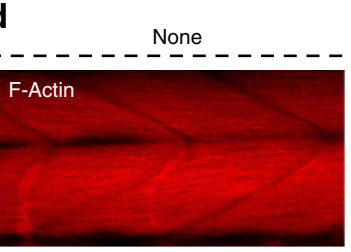

Mild
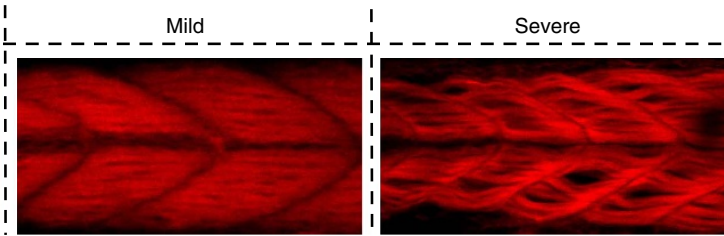

e

Control

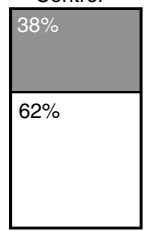

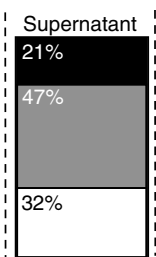

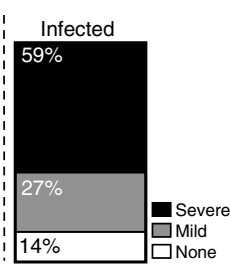

f
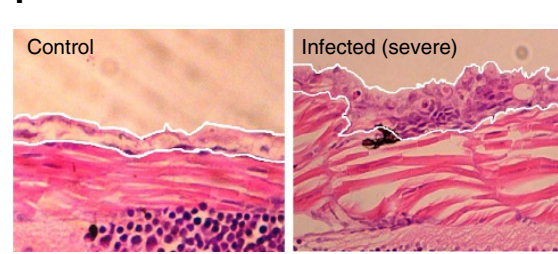

Figure 3 | Consequence of $\boldsymbol{B d}$ infection on zebrafish larvae host tissue. (a) Zebrafish larvae bath water was inoculated with low ( $<200$ zsp per $\mu \mathrm{l}$ ) dose $B d$ zoospores and incubated for 72 h.p.i., then labelled with calcoluor white (CFW; for chitin, green) and evans blue (EB; for tissue damage, red), and visualized by confocal microscopy. Images taken at $\times 40$, maximum intensity projection of Z-stack shown here. Cartoon depicts imaged region. Representative images with insets highlight colocalization of CFW-labelled punctae with EB positive tissue damage. Scale bars, $50 \mu \mathrm{m}$. See also Supplementary Fig. 3a for more examples. (b) Enumeration of apoptotic cells from larvae whose bath water was inoculated with mTGhL plate washings (control, open circles) or low (filled circles) dose Bd zoospores and incubated for 72 h.p.i., then fixed and labelled with TUNEL. Each point represents an individual larva. Data pooled from three experiments, using $n=3$ per treatment. Mean \pm s.e.m. are shown. Significance testing performed using Mann-Whitney test (two tailed), ${ }^{\star \star} P<0.01$. (c) Zebrafish larvae bath water was inoculated with high dose Bd zoospores and incubated for 72 h.p.i., then fixed for electron microscopy (EM). 1, Sloughing necrotic cell. 2, sloughed necrotic cell. Scale bars, $1 \mu \mathrm{m}$. See also Supplementary Fig. $3 \mathrm{~d}$ for EM images of larvae treated with control. (d) Zebrafish larvae bath water was inoculated with control, high ( $>200 \mathrm{zsp}$ per $\mu$ l) dose $B d$ zoospore supernatant or high dose $B d$ zoospores and incubated for 72 h.p.i., then fixed, labelled with phalloidin (for F-Actin; red) and visualized by confocal microscopy. Images taken at $40 \mathrm{X}$, maximum intensity projection of Z-stack shown here. Representative images of larva with no, mild or severe muscle degeneration are shown here.

(e) Proportion of larvae with no (white), mild (grey) or severe (black) muscle degeneration when prepared as in d. (f) Zebrafish larvae bath water was inoculated with control or high dose Bd zoospores and incubated for 72 h.p.i. Representative longitudinal histological sections shown here. White outline highlights skin of larvae, which in infected image shows hyperplasia of epithelial cells with mature and discharged sporangia.

$B d$-infected zebrafish larvae show a similar phenotype in vivo, TUNEL staining for apoptotic cells was performed. Infected larvae showed a $3.0 \pm 0.4$-fold increase in number of TUNELpositive apoptotic cells compared to controls (Fig. 3b). Apoptotic cells were mostly observed around fin edges and on the surface of larvae (Supplementary Fig. 3c), thus were likely to be epithelial cells where keratin is highly expressed ${ }^{32}$. Transmission electron microscopy showed necrotic epithelial cells sloughing off in infected larvae, as compared to healthy skin cells in control larvae (Fig. 3c; Supplementary Fig. 3d). Together, these results strongly suggest that $B d$ is damaging host tissue during infection of zebrafish larvae.
By 72 h.p.i., infected larvae showed disruption of clear muscle striations (Supplementary Fig. 2a). To further investigate this phenotype, larvae were fluorescently labelled with phalloidin to visualize actin filaments in vivo. Infected larvae showed striking muscle degeneration (Fig. 3d). Severity of muscle degeneration was classified into three categories based on the number of key phenotypes larval muscle fibres possessed, including loose packing, thinning, crumpling and deep tissue degeneration (Supplementary Fig. 3e). Using this classification system, high-dose-infected larvae show a higher percentage of severe muscle degeneration compared to $B d$ zoospore supernatant treated larvae, and both of these treatments show a higher 
percentage of severe muscle degeneration compared to controls (Fig. 3e). These results indicate that muscle degeneration may, at least in part, be the result of toxins secreted by $B d$ following infection. Histopathology on infected larvae also showed hyperplasia of skin where sporangia have formed, adjacent to severe muscle degeneration (Fig. 3f). These results demonstrate that $B d$ infection in zebrafish larvae is underpinned by severe tissue damage caused by secreted toxins and the infection process.

Intracellular colonization by $\boldsymbol{B} \boldsymbol{d}$ in zebrafish larvae. To determine how symptoms of infection in zebrafish larvae are related to the colonization of $B d$ on larvae skin, we labelled infected larvae at 72 h.p.i. with a novel $B d$-specific monoclonal antibody, mAb 5C4. This antibody binds to a carbohydrate epitope on an extracellular antigen produced by $B d^{33}$, labelling both $B d$ zoospores and zoosporangia with minimal background labelling in vivo (Supplementary Fig. 4a,b). Infected larvae present areas of skin harbouring the $B d$ secreted antigen (Fig. 4a). Antibody labelling also colocalizes with blisters and actin reorganization on infected larvae, showing disruption of host tissue in response to $B d$ infection (Fig. 4a,b, Supplementary Fig. 4c). These results are consistent with the pattern of CFW-labelled punctae described above, indicating that $\mathrm{mAb} 5 \mathrm{C} 4$ is suitable for highly specific, in-depth analysis of the $B d$ infection process. Indeed, various stages of $B d$ infection can be visualized in infected larvae by 72 h.p.i. (Fig. 4c), including germ-tubes invading epidermal cells, encysting zoospores and intracellular zoosporangia amongst hyperplastic epithelial cell build-ups. These results show that zebrafish larvae undergo an infection process similar to that of amphibians, and that $B d$ infection of zebrafish can be powerful model system to study the invasion and proliferation of $B d$ in vivo.

\section{Discussion}

Our study shows that $B d$ is able to infect and multiply on zebrafish larvae treated with antibiotics in a dose dependent manner that mimics the process of infection seen in amphibians. This demonstration of a non-amphibian vertebrate host being infected by $B d$ widens the host range previously known to be exploited by this hypervirulent chytrid lineage. Using a $B d$ monoclonal antibody (mAb 5C4), we were able to image the different stages of $B d$ infection with unprecedented resolution in vivo. Collectively, these results validate zebrafish larvae as a powerful aquatic model system within which these host- $B d$ interactions can be more fully explored. Furthermore, our observations that treating zebrafish with antibiotics results in higher burdens of infection highlight the use of probiotic bacteria to combat $B d$ infection ${ }^{34}$.

Although the specific host factors necessary for $B d$ infection remain to be discovered, $B d$ is commonly found to parasitize the keratinized tissue of both amphibian and non-amphibian hosts $8,9,35,36$. Consistent with these observations, we found $B d$ parasitizing zebrafish larvae structures known to express high levels of keratin, such as the edges of the caudal $\mathrm{fin}^{32}$, where we observed fin erosion, tissue damage and apoptotic cells. These observations are in agreement with studies showing widespread apoptosis of amphibian skin cells in response to $B d$ infection $^{6,31}$. However, in common with many macroparasite disease systems $^{37}$, not all larvae became infected and mortality is heterogeneous among experiments (Supplementary Table 1), suggesting that there are unknown factors underlying the susceptibility of zebrafish larvae to $B d$.

Heterogeneity in susceptibility of zebrafish larvae to infection may arise from a number of intrinsic and extrinsic factors, investigation of which will likely prove to be fruitful areas of future research using this model. Intrinsic factors include host immune responses and their underlying genetic determinants. Testing the expression of key inflammatory components caspase-1, interleukin $1 \beta(i l 1 \beta)$ and tumour necrosis factor $\alpha$ $(t n f \alpha)$ on $B d$-infected zebrafish larvae cDNA showed no significant difference from controls (Supplementary Fig. 5a). This result is consistent with data from other studies highlighting a complex, immunosuppressive effect on the host immune system that is possibly caused by proteases released by zoospores on infection and by maturing sporangia within the infected epidermis $^{6,38}$. As innate immune mechanisms are thought to be highly conserved from zebrafish to $\operatorname{man}^{25,39}$, the zebrafish response to $B d$ infection is likely to be similar to their amphibian counterparts. The variable expression of inflammatory markers in infected zebrafish may explain some of the heterogeneity that underlies the intensity of parasitism and mortality across our experiments. Owing to destructive sampling of whole larvae for either pathogen DNA or zebrafish RNA, we could not determine associations between the intensity of infection and expression of immunity markers. A future refinement of the model should include the development of an RNA-based marker for $B d$ so that concurrent associations between the intensity of parasitism and onset or type of immunity can be determined. Moreover, the natural translucency of zebrafish larvae enables non-invasive in vivo imaging of individual cells and $B d$-leukocyte interactions at high resolution throughout the organism ${ }^{22,24,40}$. Strikingly, the major pathogenic events that lead to chytridiomycosis in amphibians ${ }^{29}$, such as hyperplasia of epithelial cells, apoptosis of skin cells and sloughing of infected cells are faithfully reproduced in our zebrafish model. Exploiting this, we have for the first time the ability to examine the biogenesis, architecture, coordination and resolution of the innate immune response to $B d$ in vivo.

Toxins have emerged as critical molecular determinants of tissue damage during fungal infection ${ }^{5}$. We observe that larvae infected with live $B d$ show a greater extent of muscle degeneration when compared to larvae treated with $B d$ zoospore supernatant, and we propose that toxins secreted after sporangia have established in larvae skin play a role in this phenotype. Indeed, studies have shown that $B d$ zoosporangia (in comparison to zoospores) show higher expression levels of genes involved in metabolism and pathogenicity, for example, the carbohydratebinding module family 18 (CBM18), M36 metalloproteases and crinkler-like virulence effectors ${ }^{41-44}$. Using the breadth of fluorescent probes available for zebrafish larvae, our $B d$ infection model will greatly enable the in vivo investigation of known and unknown pathogenicity factors.

Extrinsic factors, including the virulence of the zoospore suspension, can influence the heterogeneity of infection phenotypes including mortality. Other factors that can influence the outcome of the host-pathogen interactions include the zebrafish microbiome and free-living aquatic predators that prey on infectious $B d$ zoospores ${ }^{45}$. Bacterial flora on amphibian skin has been shown to influence the outcome of $B d$ infection, and probiotic methods to control infection are being widely explored $^{34,46,47}$. Future work should profile the zebrafish microbiome to better understand its role in governing the process of $B d$-zebrafish infection, and manipulate the larvae microbiome to dissect the role of component bacterial species in determining the probability of $B d$ infection. To explore these issues, the use of gnotobiotic zebrafish larvae would provide a refinement to the model that has been described here ${ }^{48}$. CFW labelling showed the presence of rare punctae in unexposed control fish; these punctae may signify the presence of fishassociated oomycetes (Saprolegniales) or aquatic fungi other than $B d$. The addition of low concentrations of antibiotics to zebrafish 
a

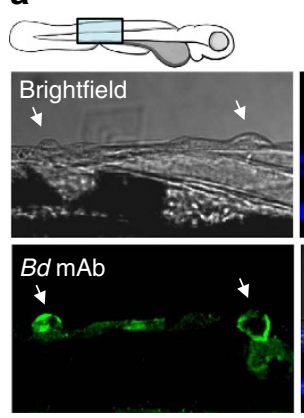

C b

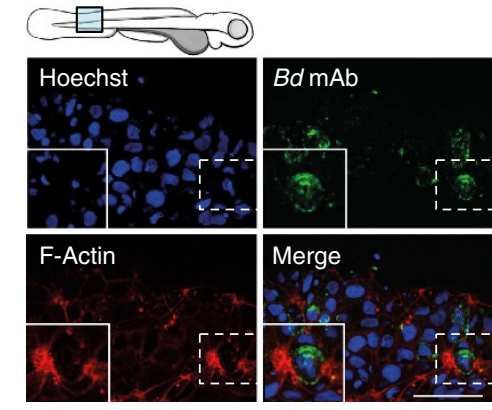

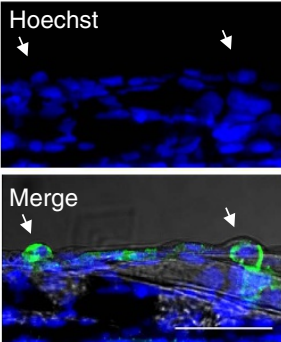

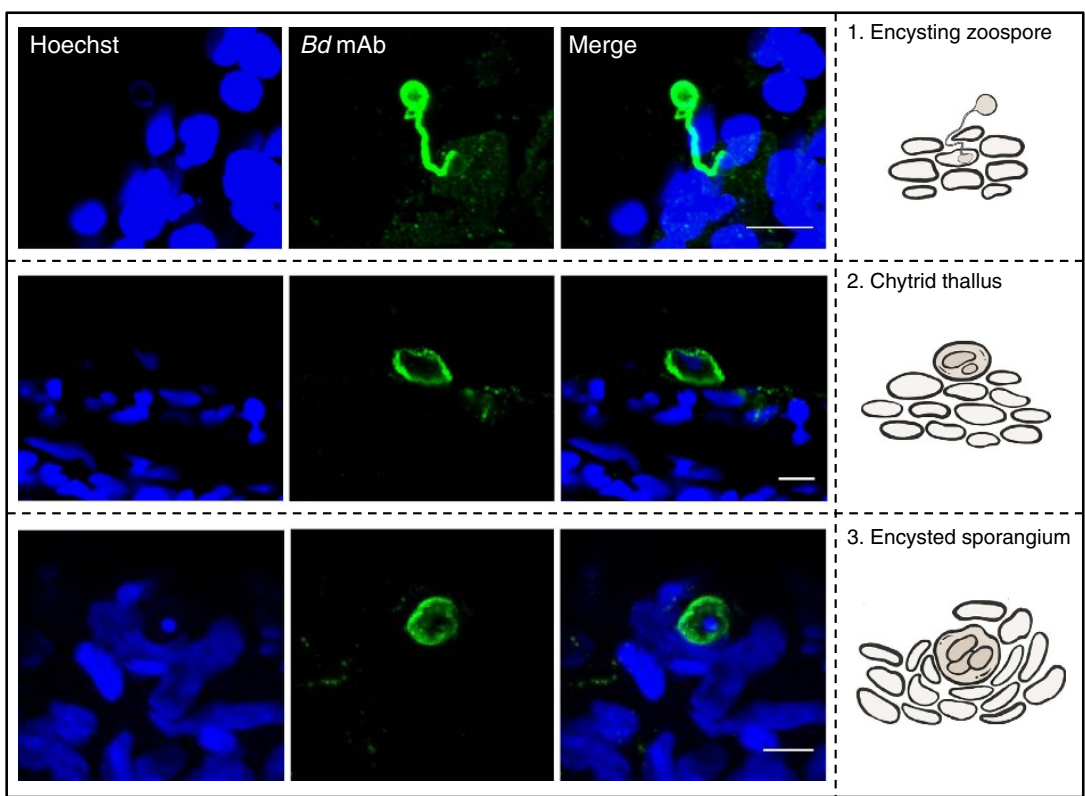

Figure 4 | Intracellular colonization by Bd in zebrafish larvae. (a) Zebrafish larvae bath water was inoculated with high ( $>200$ zsp per $\mu$ l) dose $B d$ zoospores and incubated for 72 h.p.i., then fixed and labelled with Hoechst (for DNA; blue) and mAb 5C4 (for Bd; green) for visualization by confocal microscopy. Images taken at $63 \mathrm{X}$, maximum intensity projection of $Z$-stack shown here. Cartoon depicts imaged region. Representative images with arrows highlight colocalization between $B d$ and blisters on larvae skin. Scale bar, $50 \mu \mathrm{m}$. (b) Zebrafish larvae bath water was inoculated with high dose $B d$ zoospores and incubated for 72 h.p.i., then fixed and labelled with Hoechst (for DNA; blue), mAb 5C4 (for Bd; green) and phalloidin (for F-Actin; red) for visualization by confocal microscopy. Images taken at $\times 63$, maximum intensity projection of Z-stack shown here. Cartoon depicts imaged region. Representative images with insets highlight $B d$ adjacent to host cell actin rearrangements. Scale bar, $50 \mu m$. See also Supplementary Fig. $4 c$ for host cell actin in control treated larvae. (c) Zebrafish larvae bath water was treated as in a. Images taken at $\times 40$ or $\times 63$, maximum intensity projection of Z-stack shown here. Images showing different stages of Bd invasion and infection on larvae, also depicted using cartoons in the right column. 1, rhizoid-like germ tube attached to encysting zoospore, 2, chytrid thallus growth on zebrafish larvae skin, 3, encysted sporangium amongst hyperplasic epithelial cells. Scale bars, $10 \mu \mathrm{m}$.

larvae water during infection is unlikely to remove the natural fungal fauna (the mycobiome) of the larvae in our infection model. Whether or not these commensal eukaryotes have a role in determining infection by $B d$ remains to be investigated. Finally, free-living rotifers are often found inhabiting zebrafish colonies before larvae are washed. Research has shown that aquatic microfauna can predate on the free-living infectious zoospores of $B d$, thereby lessening the probability of establishing an infection in amphibians ${ }^{45}$. The ability to co-culture rotifers with zebrafish larvae presents an opportunity to further explore the potential manipulation of aquatic microfauna as a method of increasing the resilience of ecosystems to colonization by $B d$.

Mathematical epidemiological models have shown that the highly virulent nature of $B d$ and its on-going persistence in ecosystems following host extirpations is a function of this parasite's ability to reduce the severity of density-dependent regulation through saprobic growth or parasitism of alternative, more tolerant, hosts $2,35,49,50$. Our finding that $B d$ can infect and proliferate on zebrafish larvae provides evidence that larval fish may represent an alternative reservoir of infection in nature. We tested the extent to which fish are an alternative zoonotic reservoir by infecting a second species, ornamental guppies, which represent a more mature developmental stage than 3 d.p.f. zebrafish larvae. Guppies treated with live $B d$ showed significantly increased GE of $B d$ at 5 d.p.i. compared to those treated with heat killed zoospores, however this signal was lost at 12 and 19 d.p.i. suggesting that infection is unable to persist in this host (Supplementary Fig. 1e). The increasing number of studies that have now shown conflicting evidence for the importance (or otherwise) of various non-amphibian hosts in acting as reservoirs of $B d$ is intriguing ${ }^{8-11}$. Therefore, the challenge that lies ahead is to more closely examine the biotic and abiotic factors that govern the ability of $B d$ to parasitize alternative hosts, and to understand their contribution to the dynamic nature of chytridiomycosis 
across various ecological settings. Contributing to this end, we here show that zebrafish larvae represent a tractable and powerful model aquatic system within which to explore the epidemiology and biology of this destructive chytrid.

\section{Methods}

Ethics statement. Animal experiments conducted at Imperial College or Cardiff University were performed according to the Animals (Scientific Procedures) Act 1986, and were approved by the Home Office (Project Licenses: PPL 707446 or 302876 , respectively).

Zebrafish care and maintenance. Wild-type $\mathrm{AB}$ stock was purchased from the Zebrafish International Resource Center (Eugene, OR). Eggs were obtained by placing breeding boats at the base of adult zebrafish tanks overnight, then bleached according to protocols described in The Zebrafish Book ${ }^{51}$. From 0 d.p.f. larvae were kept in petri dishes containing embryo medium (E2) without methylene blue, prepared as described in The Zebrafish Book and washed daily up to and including 3 d.p.f. Larvae were anaesthetized with $400 \mu \mathrm{g} \mathrm{ml}^{-1}$ tricane (Sigma-Aldrich) during in vivo imaging.

Growth of Batrachochytrium dendrobatidis. The strain used in this project, JEL423, was first isolated in 2004 from Phyllomedusa lemur in Panama and is the isolate from which the Broad Institute $B d$ reference genome was sequenced ${ }^{52}$. To prepare $B d$ zoospore suspension, $\mathrm{mTGhL}$ agar plates $(8 \mathrm{~g}$ typtone, $2 \mathrm{~g}$ gelatin hydrosylate, $4 \mathrm{~g}$ lactose and $10 \mathrm{~g}$ agar to $1,000 \mathrm{ml}$ of water) were seeded with actively growing $B d$ from mTGhL broth culture $(0.5 \mathrm{ml})$. These plates were sealed and incubated for $4-7$ days at $17^{\circ} \mathrm{C}$. Zoospores were collected by flooding plates with autoclaved MilliQ (MQ) water $(1 \mathrm{ml})$, rocked back and forth to dislodge zoospores, and left to stand for $10-15 \mathrm{~min}$ before collecting the supernatant; plates were flooded twice when necessary. A similar process was repeated with uninoculated control plates to obtain mTGhL plate washings (control). Zoospore concentration was determined by counting zoospores using a haemocytometer. Heat-killed $B d$ zoospores were obtained by heating zoospore suspension at $60^{\circ} \mathrm{C}$ for $10 \mathrm{~min}$ (zebrafish experiments) or $90^{\circ} \mathrm{C}$ for $15 \mathrm{~min}$ (guppy experiments). $\mathrm{Bd}$ zoospore supernatant was obtained by filtering $B d$ zoospore suspension through a $0.45 \mu \mathrm{m}$ filter to remove all zoospores. All infections were performed within $2 \mathrm{~h}$ of collecting zoospores to ensure survival of zoospores when inoculated into fish bath water ${ }^{36}$.

Zebrafish larvae infections. At 3 d.p.f. individual wild-type $A B$ zebrafish larvae were transferred into 24 -well culture plates and infected with $B d$ zoospores $(100 \mu \mathrm{l})$ in E2 without methylene blue $(1 \mathrm{ml})$, containing $1 \%$ penicillin/streptomycin prepared no more than $5 \mathrm{~h}$ before infection. Penicillin/streptomycin is commonly used to isolate $B d$ from infected amphibians ${ }^{53}$. Larvae infected with $B d$ were then incubated at $21{ }^{\circ} \mathrm{C}$. All infection experiments were incubated with the initial inoculum of $B d$, with no changes of water except in experiments conducted in Fig. 1a and Supplementary Fig. 1a where water was changed at 24 h.p.i. All experiments were terminated at 72 h.p.i. unless stated otherwise. Doses are categorized as follows, low dose $=$ final $B d$ zoospore concentration $<200$ zsp per $\mu$ l; high dose $=$ final $B d$ zoospore concentration $>200$ zsp per $\mu$ l. Control larvae represent larvae treated with $100 \mu \mathrm{l}$ of mTGhL plate washings. Un-inoculated larvae represent larvae incubated in E2 only for the entire duration of the experiment.

DNA extraction and Bd qPCR. Genomic DNA from zebrafish swabs was extracted using the bead-beating protocol published by Boyle et al. ${ }^{26}$ and adapted for zebrafish larvae as follows. Anaesthetized larvae at 1, 24, 48, 72 and/or 96 h.p.i. together with $10 \mu \mathrm{l}$ of their infected bath water were transferred into $1.5 \mathrm{ml} \mathrm{screw}$ top centrifuge tubes containing $50 \mu \mathrm{l}$ of PrepMan Ultra (Applied Biosystems) and $30-40 \mathrm{mg}$ of $0.5 \mathrm{~mm}$ silica beads. Note that in time-course experiments, samples containing larvae and infected bath water were frozen at $-20^{\circ} \mathrm{C}$ until 96 h.p.i., when extraction was performed on all samples. Samples from dose dependent studies were also extracted in parallel. Larvae were homogenized for $60 \mathrm{~s}$ in a mini beadbeater, and then centrifuged for $30 \mathrm{~s}$ at $1.3 \times 10^{4}$ r.p.m. in a microfuge. Homogenization and centrifugation was repeated twice. Samples were heated to $100^{\circ} \mathrm{C}$ in a heat block, cooled for $2 \mathrm{~min}$, then centrifuged for $3 \mathrm{~min}$ at $1.3 \times 10^{4}$ r.p.m. before collection of the supernatant. This process was repeated with $10 \mu \mathrm{l}$ of infected bath water alone as a control for each sample, and with control treated larvae and bath water as a control for the experiment. A 1/10 dilution in autoclaved MQ water of all supernatants were made before freezing extracted samples and dilutions at $-20^{\circ} \mathrm{C}$, kept for a maximum of $48 \mathrm{~h}$ post extraction.

Guppy DNA was extracted from samples suspended in 100\% ethanol using DNeasy Blood and Tissue Kit (Qiagen), following the manufacturer's protocol. Extracted DNA samples were used without dilution and kept at $-20^{\circ} \mathrm{C}$.

Real-time Taqman-qPCR assays to amplify $B d$ DNA were performed with Applied Biosystems 7300 using $25 \mu \mathrm{l}$ reactions containing 2xTaqman Master Mix (Applied Biosystems), primers 5.8S (5'-AGCCAAGAGATCCGTTGTCAAA-3') and ITS-1 (5'-CCTTGATATAATACAGTGTGCCATATGTC- $3^{\prime}$ ), TaqMan MGB probe (6FAM 5'-CGAGTCGAACAAAAT-3' MGBNFQ) ${ }^{26}$. Bovine serum albumin (BSA) at $400 \mathrm{ng}^{-1} \mathrm{l}^{-1}$ was included to increase the yield of the qPCR. Quantifications were performed on duplicate wells. Each 96-well assay plate included standard reactions containing $B d$ DNA at 1,000, 100, 10 and 1 genomic equivalents (GE) as well as negative controls containing no DNA template. Samples with greater than $0.1 \mathrm{GE}$ were considered positive for $B d$.

To eliminate background upregulation due to residual $B d$ DNA in zebrafish larvae bath water, data showing $\mathrm{GE}$ of $B d$ on zebrafish was normalized using the following formula for all qPCR figures (except in Fig. 1a and Supplementary Fig. 1b where bath water was changed at 24 h.p.i.): $\mathrm{GE}$ of $B d$ on zebrafish $=$ (larvae homogenized with $10 \mu \mathrm{l}$ of its infected bath water $)-(10 \mu \mathrm{l}$ infected bath water alone).

Histologic sections. Zebrafish larvae at 72 h.p.i. were fixed with $4 \%$ paraformaldehyde overnight at $4{ }^{\circ} \mathrm{C}$. Larvae were washed three times in PBS and mounted in $1 \%$ agarose. The agarose was dehydrated in a series of ethanol from 70 to $100 \%$ and then in $100 \%$ xylene and embedded in paraffin. Longitudinal sections of tail were stained with haematoxylin and eosin. Haematoxylin and eosin-stained tissues were imaged with an Axio Lab.A1 microscope (Carl Zeiss MicroImaging GmbH, Germany), and images acquired using an Axio Cam ERc5s colour camera and computer processed using AxioVision (Carl Zeiss MicroImaging GmbH, Germany).

Microscopy of zebrafish. In vivo imaging of $B d$ was performed by staining infected zebrafish larvae with Fluorescent Brightener 28 (F3543, Sigma), also known as calcofluor white (CFW). CFW stains chitin and cellulose and is commonly used to detect $B d$ and other fungal pathogens. Larvae were anaesthetized at 24,48 and/or 72 h.p.i. with $400 \mathrm{\mu g} \mathrm{ml}^{-1}$ tricaine and stained with $250 \mu \mathrm{g} \mathrm{ml}^{-1} \mathrm{CFW}$ prepared in autoclaved MQ water, for $20 \mathrm{~min}$ at $21^{\circ} \mathrm{C}$ in the dark, only larvae that were alive at the time of microscopy were analysed. To label apoptotic cells in $B d$-infected larvae, TUNEL staining was performed on anaesthetized 72 h.p.i. larvae using in situ Cell Death Detection Kit (Roche) following the manufacturer's protocol. Images of whole larvae were taken using a Leica M205FA fluorescence stereomicroscope, Leica Macrofluor Z16 APOA (zoom 16:1) equipped with a Leica PlanApo $2.0 \times$ lens, and a Photometric CoolSNAP HQ2 Camera.

For high resolution confocal live imaging of $B d$, infected larvae were stained at 72 h.p.i. with $1 \mathrm{mg} \mathrm{ml}^{-1} \mathrm{CFW}$ containing Evans Blue (Sigma) for $10 \mathrm{~min}$ at $21^{\circ} \mathrm{C}$ in the dark. Evans Blue is a stain used to detect myofibre tissue damage ${ }^{54}$. Larvae were positioned in $35 \mathrm{~mm}$ glass bottom dishes (MatTek) and covered with $1 \%$ low-melting-point agarose. The immobilized larvae were subsequently covered with E2 (2 ml) containing tricaine.

For antibody labelling, anaesthetized zebrafish larvae were fixed overnight at $4{ }^{\circ} \mathrm{C}$ in $4 \%$ paraformaldehyde, washed for $3 \times 5 \mathrm{~min}$ in $\mathrm{PBS}+0.4 \%$ triton, then washed for $1 \times 20 \mathrm{~min}$ in PBS $+1 \%$ triton to permeabilize larvae. Anti- $B d \mathrm{mAb}$ $5 \mathrm{C} 4$ tissue culture supernatant $+0.1 \%$ sodium Azide (primary antibody) was applied to larvae overnight at $4{ }^{\circ} \mathrm{C}$. Larvae were washed for $4 \times 15 \mathrm{~min}$ in PBS $+0.1 \%$ Tween. GFP anti-mouse (to label for $B d$ ) and phalloidin (to label for filamentous actin) were diluted $1 / 200$ in PBS and applied to larvae overnight at $4{ }^{\circ} \mathrm{C}$. Larvae were washed for $4 \times 15 \mathrm{~min}$ in PBS $+0.1 \%$ Tween. Nuclei were stained with Hoechst diluted 1/500 in PBS, and applied to larvae for $10 \mathrm{~min}$. Larvae were then washed again for $4 \times 15 \mathrm{~min}$ in PBS $+0.1 \%$ Tween. Fluorescently labelled larvae were cleared by progressive transfer to $80 \%$ glycerol. Larvae were positioned in $35 \mathrm{~mm}$ glass bottom dishes and imaged using a Zeiss LSM 710 confocal microscope.

Images were processed using ImageJ software version 10.2 (ref. 55). To measure fin length, images of individual larva were each spatially calibrated using 'set scale' function (Fig. 2a; Supplementary Fig. 2c). CFW-labelled punctae and TUNEL positive apoptotic cells were also counted using this software (Figs $2 \mathrm{~d}$ and $3 \mathrm{~b}$ ). To categorize severity of muscle degeneration, larvae were ranked by two observers based on the number of phenotypes larvae possessed when labelled with phalloidin (as described in Supplementary Fig. 3e), none $=0$, mild $=$ up to 2 and severe $=3$ or more (Fig. 3e).

Electron microscopy. For ultrastructure analyses zebrafish larvae at 72 h.p.i. were fixed in $0.5 \%$ glutaraldehyde in $200 \mathrm{nM}$ sodium cacodylate buffer for $2 \mathrm{~h}$, washed in buffer and secondarily fixed in reduced $1 \%$ osmium tetroxide and $1.5 \%$ potassium ferricyanide for $60 \mathrm{~min}$. The samples were washed in distilled water and stained overnight at $4{ }^{\circ} \mathrm{C}$ in $0.5 \%$ magnesium uranyl acetate, washed in distilled water and dehydrated in graded ethanol, infiltrated with propylene oxide and then graded Epon/PO mixtures until final embedding in full Epon resin in coffin moulds (allowing different orientations) and polymerized at $56^{\circ} \mathrm{C}$ overnight. Semi-thin survey sections were cut and stained, final ultrathin sections (typically $50-70 \mathrm{~nm}$ ) and serial sections were collected on Formvar coated slot grids then stained with Reynold's lead citrate and examined in a FEI Tecnai electron microscope with CCD camera image acquisition. 
Guppy (Poecilia reticulata) infections. Adult ornamental guppies (Poecilia reticulata) purchased from a commercial supplier were maintained in glass aquaria with suitable refugia containing $50 \mathrm{l}$ of dechlorinated water at $26( \pm 0.5){ }^{\circ} \mathrm{C}$ and $12 \mathrm{~h}$ light:12 $\mathrm{h}$ dark cycle. Fish were kept in mixed sex (1 male: 16 females) stocks and fed ad libitum commercial tropical fish flakes and freshly hatched Artemia naupli. Fry were collected twice daily from breeding tanks and transferred to plastic jars containing $800 \mathrm{ml}$ dechlorinated water. Fry were maintained in shoals of five per jar under the same temperature and lighting conditions in a temperaturecontrolled incubator until the start of the experiment. Seven days before first inoculation, guppies were acclimated to $16^{\circ} \mathrm{C}$ over $24 \mathrm{~h}$. These juveniles were aged between 14 and 21 days at time of first inoculation, fed daily and maintained at $16^{\circ} \mathrm{C}$ for the duration of experiment. Each group of fish was transferred to a glass crystalizing dish containing $50 \mathrm{ml}$ of dechlorinated water and either $3 \times 10^{6}$ live or heat-killed $B d$ zoospores. After $3 \mathrm{~h}$, guppies and zoospore solutions were gently poured into plastic jars containing $750 \mathrm{ml}$ of dechlorinated water. After $24 \mathrm{~h}$, water was completely changed in each jar. The inoculation process was repeated 48 and $96 \mathrm{~h}$ after the initial dose. Guppies were monitored for a total of 19 days. At 5, 9, and 19 days post inoculation 24-33 guppies (17-23 live- $B d$ exposed, 7-10 heat-killed exposed) were killed and preserved in $100 \%$ ethanol for subsequent qPCR analysis.

RNA extraction and host real-time qPCR. Total RNA was extracted from three anaesthetized larvae per sample at 72 h.p.i. using RNAqueous-Micro Total RNA Isolation Kit (Ambion), following the manufacturer's protocol, $\mathrm{cDNA}$ was obtained using QuantiTect Reverse Transcription Kit (Qiagen) from $1 \mu \mathrm{g}$ of RNA per reaction. Quantitative PCR was then performed on a Rotor-GeneQ (Qiagen) using SYBR Green PCR Master Mix (Applied Biosystems). Primers used include caspase-1 (FW-5'-CTCCATGCAGCCAGCAATTT- $3^{\prime}$ and RV-5'-GCAAGGCCAG TCGTTTTCTG- $\left.3^{\prime}\right), i l 1 \beta$ and tnf $\alpha$ from Stockhammer et al. ${ }^{56}$ Quantifications were performed on duplicate wells. To normalize cDNA amounts, we used the housekeeping gene gadph (FW-5'-TGGGCCAATGAAGGGAATTCTGGGAT- $3^{\prime}$ and RV-5'-TAACAGGTCAGCAACACGATGGCT- $3^{\prime}$ ) and analysed results via the $2^{-\Delta \Delta C \mathrm{~T}}$ method $^{57}$. For example, in the case of $\operatorname{tnf\alpha }, 2^{-\Delta \Delta \mathrm{CT}}$ is calculated in three steps: (1) change in cycle threshold (CT) value of tnf $\alpha$ compared to housekeeping gene $\left(\Delta \mathrm{CT}_{\text {tnfx }}\right)=\mathrm{CT}_{\text {tnfox }}$ of a sample $-\mathrm{CT}_{\text {gadph }}$ of the same sample; (2) change in CT value of control against infected samples $\left(\Delta \Delta \mathrm{CT}_{\text {tnfox }}\right)=\Delta \mathrm{CT}_{\text {tnfo }}$ of controls $-\Delta \mathrm{CT}_{\text {tnfo }}$ of an infected sample; (3) calculate $2^{\Delta \Delta \mathrm{CT}}$ tnfo for each sample. These calculations were similarly performed for other cytokines and plotted in Supplementary Fig. 5a. Fold induction from all infected samples were also normalized against values from un-inoculated larvae from the same experiment.

Statistics. These data were statistically analysed with using GraphPad Prism version 7.00 for Mac OS X, GraphPad Software, La Jolla, California, USA, www.graphpad.com (ref. 58). All data were expressed as mean \pm s.e.m. Significance testing performed by log-rank (Mantel-Cox) test (Fig. 1d), unpaired Student's $t$ test (two tailed) (Figs 1a,b and 2a; Supplementary Figs 1c,e,2c and 5a) and MannWhitney test (two tailed; Figs $2 \mathrm{~d}$ and 3b; Supplementary Fig. 2e).

Data availability. The data that support the findings of this study are available from the corresponding authors on reasonable request.

\section{References}

1. Fisher, M. C., Garner, T. W. \& Walker, S. F. Global emergence of Batrachochytrium dendrobatidis and amphibian chytridiomycosis in space, time, and host. Аnnu. Rev. Microbiol. 63, 291-310 (2009).

2. Fisher, M. C. et al. Emerging fungal threats to animal, plant and ecosystem health. Nature 484, 186-194 (2012).

3. Martel, A. et al. Batrachochytrium salamandrivorans sp. nov. causes lethal chytridiomycosis in amphibians. Proc. Natl Acad. Sci. USA 110, 15325-15329 (2013)

4. Olson, D. H. et al. Mapping the global emergence of Batrachochytrium dendrobatidis, the amphibian chytrid fungus. PLoS ONE 8, e56802 (2013).

5. Martel, A. et al. Wildlife disease. Recent introduction of a chytrid fungus endangers Western Palearctic salamanders. Science 346, 630-631 (2014).

6. Fites, J. S. et al. The invasive chytrid fungus of amphibians paralyzes lymphocyte responses. Science 342, 366-369 (2013).

7. Van Rooij, P. et al. Germ tube mediated invasion of Batrachochytrium dendrobatidis in amphibian skin is host dependent. PLoS ONE 7, e41481 (2012).

8. McMahon, T. A. et al. Chytrid fungus Batrachochytrium dendrobatidis has nonamphibian hosts and releases chemicals that cause pathology in the absence of infection. Proc. Natl Acad. Sci. USA 110, 210-215 (2013).

9. Garmyn, A. et al. Waterfowl: potential environmental reservoirs of the chytrid fungus Batrachochytrium dendrobatidis. PLoS ONE 7, e35038 (2012).

10. Shapard, E. J., Moss, A. S. \& San Francisco, M. J. Batrachochytrium dendrobatidis can infect and cause mortality in the nematode Caenorhabditis elegans. Mycopathologia 173, 121-126 (2012).
11. Betancourt-Roman, C. M., O’Neil, C. C. \& James, T. Y. Rethinking the role of invertebrate hosts in the life cycle of the amphibian chytridiomycosis pathogen. Parasitology 143, 1723-1729 (2016).

12. Khosravi, A. \& Mazmanian, S. K. Disruption of the gut microbiome as a risk factor for microbial infections. Curr. Opin. Microbiol. 16, 221-227 (2013).

13. Jani, A. J. \& Briggs, C. J. The pathogen Batrachochytrium dendrobatidis disturbs the frog skin microbiome during a natural epidemic and experimental infection. Proc. Natl Acad. Sci. USA 111, E5049-E5058 (2014).

14. Kueneman, J. G. et al. The amphibian skin-associated microbiome across species, space and life history stages. Mol. Ecol. 23, 1238-1250 (2014).

15. Rebollar, E. A. et al. Skin bacterial diversity of Panamanian frogs is associated with host susceptibility and presence of Batrachochytrium dendrobatidis. ISME J. 10, 1682-1695 (2016).

16. Fites, J. S., Reinert, L. K., Chappell, T. M. \& Rollins-Smith, L. A. Inhibition of local immune responses by the frog-killing fungus Batrachochytrium dendrobatidis. Infect. Immun. 82, 4698-4706 (2014).

17. McMahon, T. A. et al. Amphibians acquire resistance to live and dead fungus overcoming fungal immunosuppression. Nature 511, 224-227 (2014).

18. Ramsey, J. P., Reinert, L. K., Harper, L. K., Woodhams, D. C. \& Rollins-Smith, L. A. Immune defenses against Batrachochytrium dendrobatidis, a fungus linked to global amphibian declines, in the South African clawed frog, Xenopus laevis. Infect. Immun. 78, 3981-3992 (2010).

19. Ribas, L. et al. Expression profiling the temperature-dependent amphibian response to infection by Batrachochytrium dendrobatidis. PLoS ONE 4, e8408 (2009).

20. Rollins-Smith, L. A., Ramsey, J. P., Pask, J. D., Reinert, L. K. \& Woodhams, D. C. Amphibian immune defenses against chytridiomycosis: impacts of changing environments. Integr. Comp. Biol. 51, 552-562 (2011).

21. Voyles, J., Rosenblum, E. B. \& Berger, L. Interactions between Batrachochytrium dendrobatidis and its amphibian hosts: a review of pathogenesis and immunity. Microb. Infect. 13, 25-32 (2011).

22. Kanther, M. \& Rawls, J. F. Host-microbe interactions in the developing zebrafish. Curr. Opin. Immun. 22, 10-19 (2010).

23. Lieschke, G. J. \& Currie, P. D. Animal models of human disease: zebrafish swim into view. Nat. Rev. Genet. 8, 353-367 (2007).

24. Meijer, A. H. \& Spaink, H. P. Host-pathogen interactions made transparent with the zebrafish model. Curr. Drug Targets 12, 1000-1017 (2011).

25. Renshaw, S. A. \& Trede, N. S. A model 450 million years in the making: zebrafish and vertebrate immunity. Dis. Model. Mech. 5, 38-47 (2012).

26. Boyle, D. G., Boyle, D. B., Olsen, V., Morgan, J. A. \& Hyatt, A. D. Rapid quantitative detection of chytridiomycosis (Batrachochytrium dendrobatidis) in amphibian samples using real-time Taqman PCR assay. Dis. Aquat. Organ 60, 141-148 (2004).

27. Hyatt, A. D. et al. Diagnostic assays and sampling protocols for the detection of Batrachochytrium dendrobatidis. Dis. Aquat. Organ 73, 175-192 (2007).

28. Berger, L. et al. Chytridiomycosis causes amphibian mortality associated with population declines in the rain forests of Australia and Central America. Proc. Natl Acad. Sci. USA 95, 9031-9036 (1998).

29. Daszak, P. et al. Emerging infectious diseases and amphibian population declines. Emerg. Infect. Dis. 5, 735-748 (1999).

30. Berger, L., Hyatt, A. D., Speare, R. \& Longcore, J. E. Life cycle stages of the amphibian chytrid Batrachochytrium dendrobatidis. Dis. Aquat. Organ $\mathbf{6 8 ,}$ 51-63 (2005).

31. Brutyn, M. et al. Batrachochytrium dendrobatidis zoospore secretions rapidly disturb intercellular junctions in frog skin. Fungal Genet. Biol. 49, 830-837 (2012).

32. Martorana, M. L. et al. Zebrafish keratin 8 is expressed at high levels in the epidermis of regenerating caudal fin. Int. J. Dev. Biol. 45, 449-452 (2001)

33. Dillon, M. J. et al. Tracking the amphibian pathogens Batrachochytrium dendrobatidis and Batrachochytrium salamandrivorans by using a highly specific monoclonal antibody and lateral-flow technology. Microbial. Biotech. 10, 381-394 (2017).

34. Harris, R. N. et al. Skin microbes on frogs prevent morbidity and mortality caused by a lethal skin fungus. ISME J. 3, 818-824 (2009).

35. Kilburn, V. L., Ibanez, R. \& Green, D. M. Reptiles as potential vectors and hosts of the amphibian pathogen Batrachochytrium dendrobatidis in Panama. Dis. Aquat. Organ 97, 127-134 (2011).

36. Piotrowski, J. S., Annis, S. L. \& Longcore, J. E. Physiology of Batrachochytrium dendrobatidis, a chytrid pathogen of amphibians. Mycologia 96, 9-15 (2004).

37. Koprivnikar, J. et al. Macroparasite infections of amphibians: what can they tell us? EcoHealth 9, 342-360 (2012).

38. Rosenblum, E. B. et al. A molecular perspective: biology of the emerging pathogen Batrachochytrium dendrobatidis. Dis. Aquat. Organ 92, 131-147 (2010).

39. Howe, K. et al. The zebrafish reference genome sequence and its relationship to the human genome. Nature 496, 498-503 (2013).

40. Mostowy, S. et al. The zebrafish as a new model for the in vivo study of Shigella flexneri interaction with phagocytes and bacterial autophagy. PLoS Pathog. 9, e1003588 (2013) 
41. Abramyan, J. \& Stajich, J. E. Species-specific chitin-binding module 18 expansion in the amphibian pathogen Batrachochytrium dendrobatidis. Mbio, 3, e00150-00112 (2012).

42. Farrer, R. A. et al. Chromosomal copy number variation, selection and uneven rates of recombination reveal cryptic genome diversity linked to pathogenicity. PLoS Genet. 9, e1003703 (2013).

43. Liu, P. \& Stajich, J. E. Characterization of the Carbohydrate Binding Module 18 gene family in the amphibian pathogen Batrachochytrium dendrobatidis. Fungal Genet. Biol. 77, 31-39 (2015).

44. Farrer, R. A. et al. Genomic innovations linked to infection strategies across emerging pathogenic chytrid fungi. Nat Commun 8, 14742 (2017).

45. Schmeller, D. S. et al. Microscopic aquatic predators strongly affect infection dynamics of a globally emerged pathogen. Curr. Biol. 24, 176-180 (2014).

46. Becker, M. H. et al. Towards a better understanding of the use of probiotics for preventing chytridiomycosis in Panamanian golden frogs. EcoHealth 8 , 501-506 (2011).

47. Bletz, M. C. et al. Mitigating amphibian chytridiomycosis with bioaugmentation: characteristics of effective probiotics and strategies for their selection and use. Ecol. Lett. 16, 807-820 (2013).

48. Pham, L. N., Kanther, M., Semova, I. \& Rawls, J. F. Methods for generating and colonizing gnotobiotic zebrafish. Nat. Protoc. 3, 1862-1875 (2008).

49. Briggs, C. J., Knapp, R. A. \& Vredenburg, V. T. Enzootic and epizootic dynamics of the chytrid fungal pathogen of amphibians. Proc. Natl Acad. Sci. USA 107, 9695-9700 (2010).

50. Mitchell, K. M., Churcher, T. S., Garner, T. W. \& Fisher, M. C. Persistence of the emerging pathogen Batrachochytrium dendrobatidis outside the amphibian host greatly increases the probability of host extinction. Proc. R Soc. Med. 275, 329-334 (2008).

51. Westerfield, M. The Zebrafish book: A Guide for the Laboratory Use of Zebrafish (Danio Rerio) 4th edn (University of Oregon Press, 2000).

52. Lips, K. R. et al. Emerging infectious disease and the loss of biodiversity in a Neotropical amphibian community. Proc. Natl Acad. Sci. USA 103, 3165-3170 (2006).

53. Longcore, J. E. Recognizing, isolating, and culturing Batrachochytrium dendrobatidis from amphibians (Maine Chytrid Laboratory, 2004).

54. Hamer, P. W., McGeachie, J. M., Davies, M. J. \& Grounds, M. D. Evans Blue Dye as an in vivo marker of myofibre damage: optimising parameters for detecting initial myofibre membrane permeability. J. Anat. 200, 69-79 (2002).

55. ImageJ (Rasband, W.S., US National Institute of Health, 1997).

56. Stockhammer, O. W., Zakrzewska, A., Hegedus, Z., Spaink, H. P. \& Meijer, A. H. Transcriptome profiling and functional analyses of the zebrafish embryonic innate immune response to Salmonella infection. J. Immunol. 182, 5641-5653 (2009).

57. Livak, K. J. \& Schmittgen, T. D. Analysis of relative gene expression data using real-time quantitative PCR and the $2^{-\Delta \Delta C}$ T method. Methods 25, 402-408 (2001).

58. GraphPad v. 7 (GraphPad Software Inc., 1984).

\section{Acknowledgements}

We thank Susanne Herbst for providing reagents, Lorraine Lawrence for performing histopathology on larvae, Willow Smallbone for guppy husbandry and to the members of the Mostowy and Fisher labs for zebrafish husbandry, $B d$ preparations, and helpful discussions. N.L. is supported by the Lister Institute of Preventive Medicine. J.C. and A.E. were funded by the Welsh Government and Higher Education Funding Council for Wales through the Sêr Cymru National Research Network for Low Carbon, Energy and Environment AquaWales Project. C.R.T. and M.J.D. were funded by the Leverhulme Trust. Work in the Fisher laboratory is supported by the UK Natural Environmental Research Council (NERC NE/K014455/1) and the Leverhulme Trust. Work in the Mostowy laboratory is supported by a Wellcome Trust Research Career Development Fellowship (WT097411MA) and the Lister Institute of Preventive Medicine.

\section{Author contributions}

N.L., M.J.M.M., M.C.F. and S.M. designed the research and carried out analysis. N.L., M.J.M.M., C.J.W. and M.H. performed experiments on D. rerio (zebrafish larvae) with supervision and assistance from M.C.F. and S.M. M.J.D. and C.R.T. designed the $B d \mathrm{mAb} 5 \mathrm{C} 4$ antibody, A.E. and J.C. performed all experiments on P. reticulata (ornamental guppies). N.L., M.C.F. and S.M. wrote the manuscript with input from all authors.

\section{Additional information}

Supplementary Information accompanies this paper at http://www.nature.com/ naturecommunications

Competing interests: The authors declare no competing financial interests.

Reprints and permission information is available online at http://npg.nature.com/ reprintsandpermissions/

How to cite this article: Liew, N. et al. Chytrid fungus infection in zebrafish demonstrates that the pathogen can parasitize non-amphibian vertebrate hosts. Nat. Commun. 8, 15048 doi: 10.1038/ncomms15048 (2017).

Publisher's note: Springer Nature remains neutral with regard to jurisdictional claims in published maps and institutional affiliations.

This work is licensed under a Creative Commons Attribution 4.0 International License. The images or other third party material in this article are included in the article's Creative Commons license, unless indicated otherwise in the credit line; if the material is not included under the Creative Commons license, users will need to obtain permission from the license holder to reproduce the material. To view a copy of this license, visit http://creativecommons.org/licenses/by/4.0/

(C) The Author(s) 2017 